ponent. The percentage of all hospitals reporting at least 1 calendar-month per year of data from the hospitalwide component decreased from $95 \%$ in 1986 to $31 \%$ in 1995 . During this period, use of the hospitalwide component was greater among the hospitals whose first participation in the NNIS system occurred before 1987. Interest by NNIS hospitals in the hospitalwide component decreased from 1987 to 1995. These results suggest an evolution in the way in which NNIS hospitals conduct surveillance of nosocomial infections. The increased interest in surveillance using NNIS components that allow for risk adjustment and interhospital comparison of infection rates suggests that the feasibility and interest for such data are high.

When NNIS began in the 1970s, forms were filled out by hand by infection control personnel and sent to the CDC for analyses and publication of the surveillance data. In 1986, the CDC introduced software for NNIS hospitals, so that data could be entered and sent via floppy disk to the CDC. Beginning in 1991, the CDC distributed the first NNIS Semiannual Report that included comparative data with risk-adjusted nosocomial infection rates. Beginning in 1994, the CDC incorporated these comparative data directly into the software that all NNIS hospitals use. When a hospital calculates its infection rate for an ICU or a surgical procedure, the software automatically compares the hospital's rate with the aggregated NNIS rate, giving the hospital's percentile. In addition, the software performs an appropriate statistical test to help a hospital determine if their rate differs significantly from the pooled mean. As more data are collected at the $\mathrm{CDC}$, aggregated updates are distributed to NNIS hospitals. In June 1996, the CDC implemented telecommunications facilities as a main feature of the NNIS software. This new system allows NNIS hospitals to transmit data to the CDC electronically via telephone lines, rather than by sending floppy disks. In addition, NNIS hospitals may communicate with the CDC via electronic mail, may receive computer assistance interactively, and may obtain software updates automatically.

Since the early 1990s, many US hospitals have attempted to use quantitative standards, or benchmarks, to evaluate the quality of care they provide to patients. Using these benchmarks in infection control has certain limitations, including the need for risk adjustment of infection rates. However, advances in data collection, analysis, dissemination, and technology has allowed the CDC to strengthen its role in aggregating data to evaluate quality of care in hospitals. These advances are helping to keep the CDC's NNIS system the leader in the use of quantitative standards for hospitals to evaluate their quality of care.

\section{Joint Commission Discusses Cooperative Accreditation Agreements}

The Joint Commission has begun discussions with five additional accrediting bodies considered as potential candidates for cooperative accreditation agreements. These organizations include the American Association of
Blood Banks, which accredits 2,150 transfusion centers; the Commission on Cancer, which accredits 1,405 hospitalbased cancer programs; and the Council on Accreditation of Services for Families and Children, which accredits 675 providers of social, community, and behavioral services. Initial discussions also have begun with two smaller accrediting bodies: the Foundation for Hospice and Homecare, which accredits 90 home care aid programs, and the Medical Quality Commission, which now accredits 30 prepaid medical groups and is growing.

FROM: Joint Commission on Accreditation of Healthcare Organizations. This month at the Joint Commission. Press releases for May 1996. Joint Commission Home Page: http://www.jcaho.org.

\section{Needle-Swap Programs Debated}

Although currently illegal in most states, needleexchange programs could prevent more than 11,000 new HIV infections by the end of the decade, two researchers reported at the 11th International Conference on AIDS. Dr. Peter Lurie of the Center for AIDS Prevention Studies at the University of California San Francisco and Ernest Drucker of Albert Einstein College of Medicine in New New York said that based on the results of the effectiveness of such programs, as many as 10,000 infections could have been prevented between 1987 and 1995 if clean-needle programs had been implemented. Other researchers praised the study - the first to estimate the national effect of needle exchanges - and said the figures may even underestimate the potential impact of the programs.

Needle exchange programs are controversial, because some people believe that they encourage the use of illegal drugs. Federal funds cannot be used to support the programs, and at least nine states prohibit the distribution of needles without a prescription.

FROM: Bennet A. Needle-swap programs spark lifeand-death debate. Wall Street Journal July 10, 1996;B1.

\section{Animal to Human Transplants}

In a new report from the Institute of Medicine (IOM), experts cautiously approve animal-to-human transplants, agreeing that the potential benefits of the technology outweigh the risks involved. The panel advises that human clinical trials should proceed only after a sufficient science base has been developed and federal guidelines and other safeguards have been established. The IOM committee issues its recommendations in the report "Xenograft Transplantation: Science, Ethics, and Public Policy," released July 17, 1996.

The transplantation of animal organs and tissue to humans, xenotransplantation, has grown in acceptance in recent years due to a shortage of human donors and a better understanding of the human body's rejection of foreign tissue. However, there has been concern that such transplants also may transfer infectious agents to humans and could endanger public health by introducing new diseases to the human population. 